OPEN ACCESS

Edited by: Rohini Mehta,

BioReliance, United States

Reviewed by:

Haijing Deng,

Westlake University, China

Xiaoping Zhong,

Second Affiliated Hospital of Shantou

University Medical College, China

Yong-Fa Zhang,

Fudan University, China

*Correspondence:

Shao-Hua Li

lishaoh@sysucc.org.cn

Rong-Ping Guo

guorp@sysucc.org.cn

${ }^{\dagger}$ These authors have contributed equally to this work

Specialty section:

This article was submitted to

Gastrointestinal Cancers,

a section of the journal

Frontiers in Oncology

Received: 16 October 2020

Accepted: 26 January 2021

Published: 25 February 2021

Citation:

Mei J, Tang Y-H, Wei W, Shi M, Zheng L, Li S-H and Guo R-P (2021)

Hepatic Arterial Infusion Chemotherapy Combined With PD-1 Inhibitors Plus Lenvatinib Versus PD-1 Inhibitors Plus Lenvatinib for Advanced Hepatocellular Carcinoma.

Front. Oncol. 11:618206 doi: 10.3389/fonc.2021.618206

\section{Hepatic Arterial Infusion} Chemotherapy Combined With PD-1 Inhibitors Plus Lenvatinib Versus PD-1 Inhibitors Plus Lenvatinib for Advanced Hepatocellular Carcinoma

\author{
Jie Mei ${ }^{1,2,3+}$, Yu-Hao Tang ${ }^{1,2,3+}$, Wei Wei ${ }^{1,2,3}$, Ming Shi ${ }^{1,2,3}$, Lie Zheng ${ }^{2,3,4}$, \\ Shao-Hua Li $^{1,2,3^{*}}$ and Rong-Ping Guo ${ }^{1,2,3^{*}}$ \\ ${ }^{1}$ Department of Liver Surgery, Sun Yat-Sen University Cancer Center, Guangzhou, China, ${ }^{2}$ State Key Laboratory of \\ Oncology in South China, Guangzhou, China, ${ }^{3}$ Collaborative Innovation Center for Cancer Medicine, Guangzhou, China, \\ ${ }^{4}$ Department of Medical Imaging, Sun Yat-Sen University Cancer Center, Guangzhou, China
}

Background: Lenvatinib combined with programmed cell death protein-1 (PD-1) inhibitors has resulted in good survival outcomes in the treatment of unresectable hepatocellular carcinoma (HCC). Hepatic artery infusion chemotherapy (HAIC) has also attracted attention due to its high response rates and favorable survival for advanced HCC patients. The present study aimed to compare the efficacy of HAIC combined with PD-1 inhibitors plus lenvatinib (HPL) and PD-1 inhibitors plus lenvatinib (PL) in patients with advanced HCC.

Methods: Between July 2018 and December 2019, patients diagnosed with advanced HCC who initially received HPL or PL treatment were reviewed for eligibility. Efficacy was evaluated according to tumor response and survival.

Results: In total, 70 patients met the criteria and were included in the present study, and they were divided into the HPL group $(n=45)$ and PL group $(n=25)$. The overall response rate (40.0 vs. $16.0 \%$, respectively; $p=0.038)$ and disease control rate $(77.6$ vs. $44.0 \%$, respectively; $p<0.001$ ) were higher in the HPL group than in the PL group. The median overall survival was 15.9 months in the HPL group and 8.6 months in the $\mathrm{PL}$ group ( $\mathrm{p}=0.0015 ; \mathrm{HR}=0.6 ; 95 \% \mathrm{Cl} 0.43-0.83$ ). The median progression-free survival was 8.8 months in the HPL group and 5.4 months in the PL group ( $p=0.0320$; $\mathrm{HR}=0.74 ; 95 \% \mathrm{Cl} 0.55-0.98)$

Conclusion: Compared to PL, HPL was associated with a significantly better treatment response and survival benefits for patients with advanced HCC.

Keywords: hepatocellular carcinoma, hepatic artery infusion chemotherapy, programmed cell death protein-1, lenvatinib, FOLFOX 


\section{INTRODUCTION}

Hepatocellular carcinoma (HCC) is one of the most common malignancies and the fourth leading cause of cancer-related deaths worldwide (1). For advanced HCC, surgical resection is inapplicable, and locoregional approaches bring little benefit (2). Lenvatinib and programmed cell death protein-1 (PD-1) inhibitors are currently well-studied and proven to bring survival benefit as first- and second-line treatment of advanced HCC (3-5). In an open-label multicenter study, lenvatinib plus pembrolizumab surprisingly showed a median overall survival (OS) of 22 months and a median progression-free survival (PFS) of 8.6 months in patients with unresectable hepatocellular carcinoma (6). In recent years, hepatic artery infusion chemotherapy (HAIC) has attracted attention due to high response rates and favorable survival for advanced HCC (7). Several randomized clinical trials have shown that HAIC combined with sorafenib yields significantly better survival compared to sorafenib monotherapy $(8,9)$. These findings imply that HAIC may have potential when combined with targeted drug therapy.

To date, no research has studied the efficacy of HAIC in combination with lenvatinib and PD-1 inhibitors. Therefore, we designed this retrospective study to compare the survival of patients with advanced HCC who received HAIC combined with lenvatinib plus PD-1 inhibitors (HPL) versus those who received lenvatinib plus $\mathrm{PD}-1$ inhibitors $(\mathrm{PL})$, aiming to provide a reference for the treatment of advanced HCC.

\section{METHODS}

This study was conducted according to the ethical guidelines of the 1975 Declaration of Helsinki. The analysis of the patient data was reviewed and approved by the Institutional Review Board and Human Ethics Committee at the Sun Yat-Sen University Cancer Center (SYSUCC; Guangzhou, China).

\section{Patients}

Between July 2018 and December 2019, the medical records of patients diagnosed with HCC who received HPL and PL treatment at the Department of Liver Surgery of SYSUCC were reviewed for eligibility. Patients were included based on the following specific criteria: a) patients were diagnosed with HCC through imaging or pathology according to the AASLD practice guidelines (10); b) no cancer-related therapies were involved before or during HPL or PL; c) patients had a tumor classification of Barcelona Clínic Liver Cancer (BCLC) B or C; d) Child-Pugh (CP) was classified as A or B; e) patients had at least two cycles of HPL or PL; f) no other malignant tumors were diagnosed; and g) complete medical and follow-up data were available. All laboratory serum test data were collected within 3 days before the initial treatment. Imaging evaluation included enhanced computed tomography (CT) or magnetic resonance imaging (MRI) examination within a week before the initial treatment.

\section{Treatment Procedure}

Lenvatinib (The UK, Eisai Europe Co. Ltd.) (8 to $12 \mathrm{mg}$ according to bodyweight) was taken orally. PD-1 inhibitors were used intravenously at the standard dose (Table S1). The first use of PD-1 inhibitors was within 7 days of initiation of lenvatinib. For the HPL group, HAIC was administered according to previously described procedures (11). Femoral artery puncture and catheterization were performed in every cycle of treatment. The FOLFOX regimen was administered via the hepatic artery as follows: 85 or $135 \mathrm{mg} / \mathrm{m}^{2}$ oxaliplatin, $400 \mathrm{mg} / \mathrm{m}^{2}$ leucovorin, and $400 \mathrm{mg} / \mathrm{m}^{2}$ fluorouracil on the first day; and $2400 \mathrm{mg} / \mathrm{m}^{2}$ fluorouracil over $46 \mathrm{~h}$. Patients received PD-1 inhibitors and lenvatinib within 3 days before or after the start of HAIC. The discontinuation of treatment depended on disease progression,

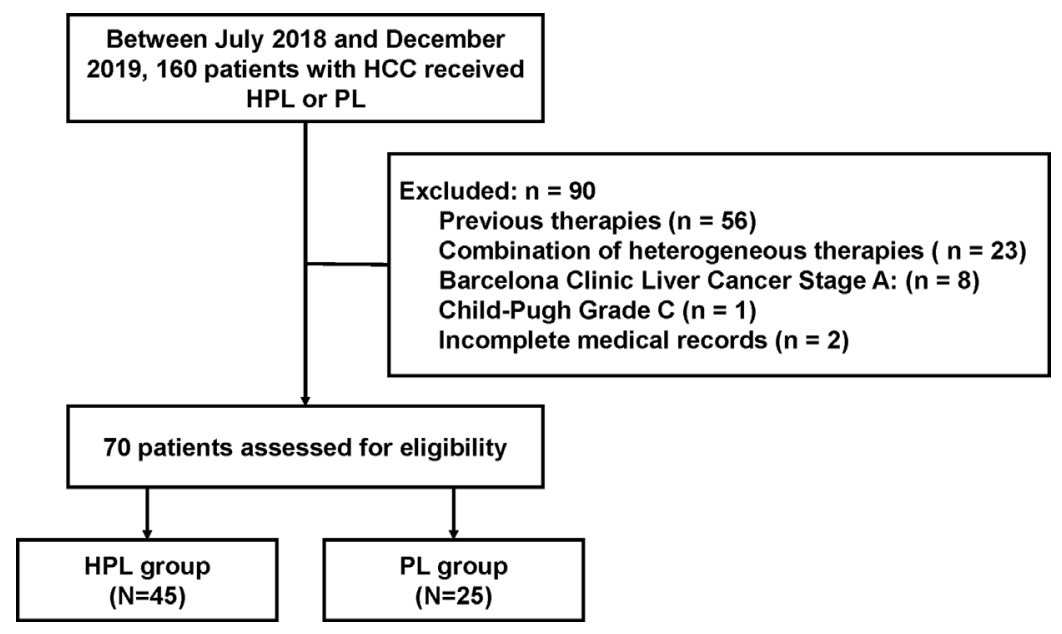

FIGURE 1 | Flow diagram summarizing the disposition process of patients. 
unacceptable toxicity, patient withdrawal of consent, or changes of treatment plan. The final follow-up ended on September 30, 2020. Enhanced CT or MRI was performed every 2 or 3 months. Routine follow-up intervals were 2 to 4 months.

\section{Diagnosis and Definitions}

Tumor response was defined as complete response (CR), partial response (PR), stable disease (SD), or progressive disease (PD) according to the modified Response Evaluation Criteria in Solid Tumors 1.1 (mRECIST) (12). Overall response rate (ORR) was calculated as the sum of CR and PR. Disease control rate (DCR) was calculated as the sum of CR, PR, and SD. Overall survival (OS) was defined as the time interval from treatment initiation to cancer-related death. Progression-free survival (PFS) was defined as the time interval from treatment initiation to progression or death. Treatment-related adverse events (AEs) were evaluated by National Cancer Institute Common Terminology Criteria for Adverse Events version 4.0.

\section{Statistical Analysis}

Categorical variables in the baseline characteristics were compared using the Pearson's $\chi 2$ test or Fisher's exact test. Variable distribution was described using mean \pm standard error (SE) for normally distributed values, and median and range were used for non-normally distributed values. Survival analysis was calculated using the Kaplan-Meier method, and differences in the survival curves were analyzed with a log-rank test. All variables with a $\mathrm{P}$ value $<0.05$ in univariate analyses were used in multivariate analyses using Cox regression models. The hazard ratio (HR) and confidence intervals (CI) were calculated. A two-tailed $\mathrm{P}$ value $<0.05$ was considered statistically significant. All data analyses were performed using SPSS 25.0 software (SPSS Inc., Chicago, IL) and GraphPad Prism (version 8.0; GraphPad, Inc.).

\section{RESULTS}

\section{Identification and Characteristics of Study Patients}

From July 2018 to December 2019, 160 patients with HCC who received HPL or PL were screened: 56 patients received previous surgery, interventional therapies, tyrosine kinase inhibitors or immune-targeted therapies; 23 patients participated in other treatments during HPL or PL; 8 patients were classified with a tumor grade of BCLC/A; 1 patient was classified as $\mathrm{CP}$ C; and 2 patients had missing sections in their medical records. Finally, a total of 70 patients who met the criteria were included in the study, and the patients were divided into the HPL group $(n=45)$ and PL group $(n=25)$. The patient characterization process is shown in Figure 1. Of note, the treatment of PD-1 inhibitors plus lenvatinib was available since July 2018 at our center.

The clinical characteristics and treatment of patients are summarized in Table 1. Most patients were classified into CP A (97.8\% in the HPL group and $88 \%$ in the PL group) and BCLC/ $\mathrm{C}(88.9 \%$ in the HPL group and $88.0 \%$ in the PL group). Two
TABLE 1 | Baseline clinical characteristics of patients.

\begin{tabular}{|c|c|c|c|}
\hline Characteristic* & HPL $(n=45)$ & PL (n=25) & $\begin{array}{c}\mathbf{P} \\
\text { value }\end{array}$ \\
\hline Age & $49.1 \pm 10.6$ & $50.1 \pm 12.3$ & 0.366 \\
\hline Gender* & & & 0.212 \\
\hline Male & $38(84.4)$ & $18(72.0)$ & \\
\hline Female & 7 (15.6) & $7(28.0)$ & \\
\hline HBV & & & 0.533 \\
\hline Negative & $8(17.8)$ & $6(24.0)$ & \\
\hline Positive & $37(82.2)$ & $19(76.0)$ & \\
\hline $\mathrm{HCV}$ & & & 1.000 \\
\hline Negative & $44(97.8)$ & 25 (100.0) & \\
\hline Positive & $1(2.2)$ & $0(0)$ & \\
\hline WBC (10E9/L) & $6.7(5.6,8.0)$ & $7.4(5.7,9.7)$ & 0.420 \\
\hline NEU (10E9/L) & $4.78(3.66,5.92)$ & $4.86(3.20,7.20)$ & 0.876 \\
\hline LYM (10E9/L) & $1.2(0.9,1.6)$ & $1.4(1.1,1.8)$ & 0.210 \\
\hline $\mathrm{HB}(\mathrm{g} / \mathrm{L})$ & $138.4 \pm 20.7$ & $137.9 \pm 29.6$ & 0.944 \\
\hline PLT (10E9/L) & $227.0(184.0,328.0)$ & $201.0(149.8,281.5)$ & 0.351 \\
\hline $\operatorname{ALT}(\mathrm{U} / \mathrm{L})$ & $45.5(34.0,74.4)$ & $57.0(37.6,89.8)$ & 0.329 \\
\hline AST (U/L) & $76.2(55.6,152.1)$ & $96.7(56.7,173.4)$ & 0.655 \\
\hline AFP (ng/ml) & $\begin{array}{c}4106.0(72.8 \\
121000.0)\end{array}$ & $767.6(23.3,21940.5)$ & 0.193 \\
\hline PIVKA-II (mAU/ml) & $\begin{array}{c}9929.0(1672.0 \\
51343.0)\end{array}$ & $\begin{array}{c}11794.5(252.3 \\
75000.0)\end{array}$ & 0.952 \\
\hline Liver cirrhosis & & & 0.143 \\
\hline Absent & $5(11.1)$ & $7(28.0)$ & \\
\hline Present & $40(88.9)$ & $18(72.0)$ & \\
\hline Child-Pugh & & & 0.127 \\
\hline A & $44(97.8)$ & $22(88.0)$ & \\
\hline B & $1(2.2)$ & $3(12.0)$ & \\
\hline Barcelona Clínic Liver & & & 1.000 \\
\hline \multicolumn{4}{|l|}{ Cancer } \\
\hline B & $5(11.1)$ & $3(12.0)$ & \\
\hline C & $40(88.9)$ & $22(88.0)$ & \\
\hline $\begin{array}{l}\text { Size of largest nodule } \\
(\mathrm{cm})\end{array}$ & $11.2 \pm 3.9$ & $10.9 \pm 4.2$ & 0.754 \\
\hline Tumor number & & & 1.000 \\
\hline Solitary & $9(20.0)$ & $5(20.0)$ & \\
\hline Multiple & $36(80.0)$ & $20(80.0)$ & \\
\hline Tumor distribution & & & 0.409 \\
\hline Uni-lobar & $17(37.8)$ & 7 (28.0) & \\
\hline Bi-lobar & $28(62.2)$ & $18(72.0)$ & \\
\hline Tumor thrombus & & & 0.237 \\
\hline Absent & $9(20.0)$ & $7(28.0)$ & \\
\hline Branch of portal vein & $20(44.4)$ & $6(24.0)$ & \\
\hline Main portal vein & $16(35.6)$ & $12(48.0)$ & \\
\hline Extrahepatic metastasis & & & 0.127 \\
\hline Absent & $30(66.7)$ & $12(48.0)$ & \\
\hline Present & 15 (33.3) & $13(52.0)$ & \\
\hline
\end{tabular}

*No (\%).

$P D-1$, programmed cell death protein-1; HPL, hepatic artery infusion chemotherapy combined with $P D-1$ inhibitors plus lenvatinb; $P L, P D-1$ inhibitors plus lenvatinib; HBV, hepatitis B virus; HCV, hepatitis C virus; WBC, white blood cell; NEU, neutrophil; $L Y M$, lymphocyte; HB, haemoglobin; PLT, blood platelet; ALT, alanine aminotransferase; AST, aspartate aminotransferase; AFP, alpha fetoprotein; PIVKA-II, protein induced by vitamin K absence or antagonist-I.

groups were comparable in the clinical characteristics, liver function, and tumor characteristics. A higher proportion of patients in the PL group had extrahepatic metastasis compared to the HPL group (52.0 vs. 33.3\%), but the difference was not statistically significant ( $p=0.127)$. In the HPL group, the cycles of PD-1 inhibitors plus lenvatinib ranged from 2 to 12 , with a median of 5 . While in the PL group, the cycles of PD-1 inhibitors plus lenvatinib ranged from 2 to 9 , with a median 
of 4. The PD-1 inhibitor categories in each group are summarized in Table S1.

\section{Survival}

The median follow-up time was 15.1 months. Patients in the HPL group had significantly better survival outcomes than those in the PL group. The 3-, 6-, and 12-month OS was 97.8, 86.7, and $67.4 \%$, respectively, in the HPL group, and 83.6, 61.8, and $29.8 \%$, respectively, in the PL group. The median OS was 15.9 months in the HPL group and 8.6 months in the PL group $(\mathrm{p}=0.0015 ; \mathrm{HR}=$ 0.6 ; 95\% CI 0.43-0.83). The 3-, 6-, and 12-PFS was 86.7, 68.9, and $43.2 \%$, respectively, in the HPL group, and $75.8,49.2$, and $15.7 \%$,
A

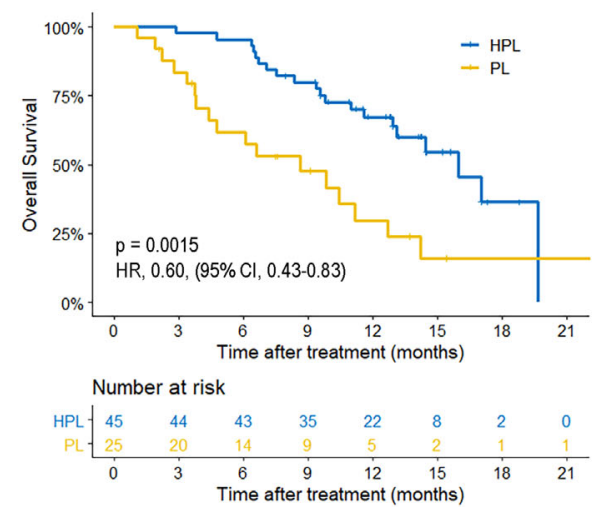

B
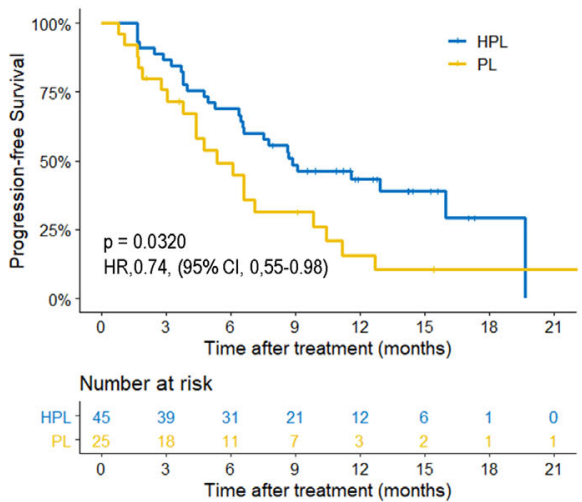

FIGURE 2 | Kaplan-Meier curves of survival outcomes of patients in the two groups. (A) Overall survival. (B) Progression-free survival. HPL, hepatic artery infusion chemotherapy combined with PD-1 inhibitors plus lenvatinib; PL, PD-1 inhibitors plus lenvatinib.

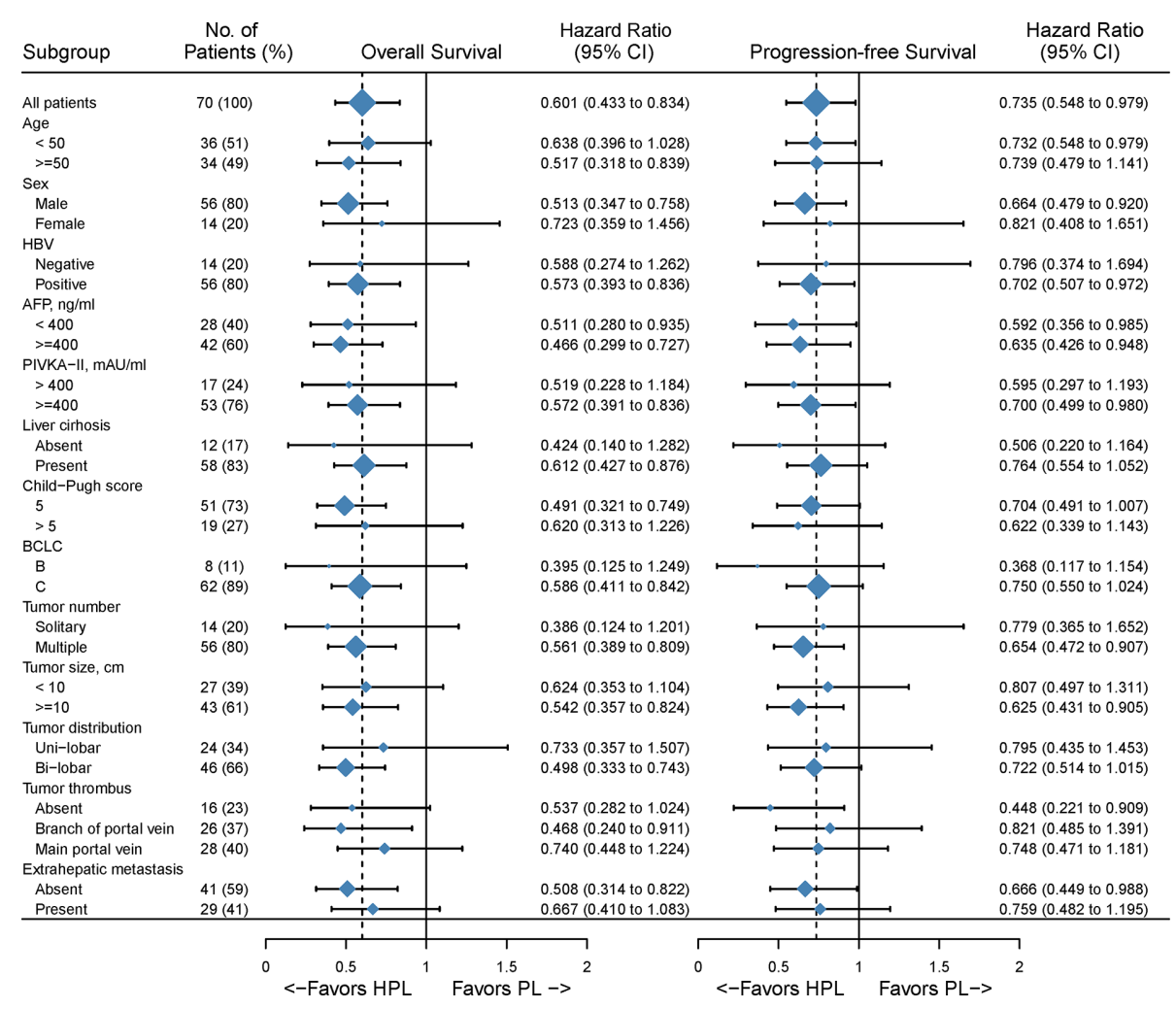

FIGURE 3 | Forest plot for overall survival of the matched cohorts of patients. HPL, hepatic artery infusion chemotherapy combined with PD-1 inhibitors plus lenvatinib; PL, PD-1 inhibitors plus lenvatinib. 
respectively, in the PL group. The median PFS was 8.8 months in the HPL group and 5.4 months in the PL group $(\mathrm{p}=0.0320$; $\mathrm{HR}=$ 0.74; 95\% CI 0.55-0.98). The survival curves are shown in Figure 2. The forest plot analysis of factors associated with OS and PFS

TABLE 2 | Summary of best response.

\begin{tabular}{lccc}
\hline Variable & HPL $(\mathbf{n}=\mathbf{4 5}) \mathbf{N o .}(\mathbf{\%})^{\mathbf{c}}$ & $\mathbf{P L}(\mathbf{n}=\mathbf{2 5}) \mathbf{N o .}(\mathbf{\%})^{\mathbf{c}}$ & P value \\
\hline Overall response $^{\mathbf{a}}$ & & & \\
Complete response & $0(0.0)$ & $0(0.0)$ & 1.000 \\
Partial response & $18(40.0)$ & $4(16.0)$ & 0.038 \\
Stable response & $20(44.4)$ & $7(28.0)$ & 0.176 \\
Progressive response & $5(11.1)$ & $6(24.0)$ & 0.156 \\
Not assessable & $2(4.4)$ & $8(32.0)$ & 0.002 \\
Overall response rate & $18(40.0)$ & $4(16.0)$ & 0.038 \\
Disease control rate & $38(77.6)$ & $11(44.0)$ & $<0.001$ \\
Intrahepatic response ${ }^{\mathbf{b}}$ & & & \\
Complete response & $0(0.0)$ & $0(0.0)$ & 1.000 \\
Partial response & $18(40.0)$ & $4(16.0)$ & 0.038 \\
Stable response & $22(48.9)$ & $9(36.0)$ & 0.298 \\
Progressive response & $3(6.7)$ & $4(16.0)$ & 0.212 \\
Not assessable & $2(4.4)$ & $8(32.0)$ & 0.002 \\
Overall response rate & $18(40.0)$ & $4(16.0)$ & 0.038 \\
Disease control rate & $40(88.9)$ & $13(52.0)$ & 0.001 \\
\hline
\end{tabular}

HPL, hepatic artery infusion chemotherapy combined with programmed cell death protein-1 inhibitors plus lenvatinb; PL, programmed cell death protein-1 inhibitors plus lenvatinib.

${ }^{a}$ Overall response included assessment of the change in tumor burden inside and outside the liver.

${ }^{b}$ fimmu.2021.619776Intrahepatic response only included assessment of the change in tumor burden inside the liver.

${ }^{c}$ Treatment response was assessed in evaluable patients. is shown in Figure 3. HPL provided a clinical benefit in patients with large, multiple HCCs, but it failed to have a survival benefit in patients with main portal vein tumor thrombus or extrahepatic metastasis.

\section{Tumor Response}

The treatment response is summarized in Table 2. Based on mRESIST, the ORR was higher in the HPL group (40.0\%) than in the PL group $(16.0 \%)(p=0.038)$. A higher DCR in both overall response (77.6 vs. $44.0 \%$; $<<0.001)$ and intrahepatic response (88.9 vs. 52.0\%; $\mathrm{p}=0.001$ ) was present in the HPL group compared to the PL group.

\section{Safety Analysis}

All AEs were evaluated as mild and manageable, and no toxicityassociated deaths occurred in the follow-up. More patients in the HPL group experienced grade 1-2 neutropenia and increased alanine aminotransferase. Only one patient experienced Grade 3 pain in the PL group. The details of the events were summarized in Table 3.

\section{Prognostic Factor Analysis}

The prognostic factors for survival are shown in Table 4. The comparison of PL to HPL was identified as an independent risk factor for both OS (HR = 3.180; 95\% CI 1.608-6.290; $\mathrm{p}=0.001$ ) and PFS $(\mathrm{HR}=2.702 ; 95 \% \mathrm{CI} 1.440-5.070 ; \mathrm{p}=0.002)$. In addition, multivariate analyses identified that $\mathrm{CP} B$ and multiple tumors were risk factors for OS and that AFP $\geq 400$ $\mathrm{ng} / \mathrm{ml}$ was a risk factor for PFS.

TABLE 3 | Treatment-related adverse events.

\begin{tabular}{|c|c|c|c|c|c|c|}
\hline Adverse events & \multicolumn{3}{|c|}{ Any grade } & \multicolumn{3}{|c|}{ Grade $3 / 4$} \\
\hline Treatment-related AEs, n (\%) & & & & $0(0)$ & $1(4.0)$ & 0.357 \\
\hline Pruritus & $3(6.7)$ & $2(8.0)$ & 1.000 & $0(0)$ & $0(0)$ & 1.000 \\
\hline Pain & $7(15.6)$ & $7(28.0)$ & 0.212 & $0(0)$ & $1(4.0)$ & 0.357 \\
\hline Fever & $12(26.7)$ & $5(20.0)$ & 0.500 & $0(0)$ & $0(0)$ & 1.000 \\
\hline Nausea & $6(13.3)$ & $1(4.0)$ & 0.408 & $0(0)$ & $0(0)$ & 1.000 \\
\hline Decreased appetite & $8(17.8)$ & $2(8.0)$ & 0.314 & $0(0)$ & $0(0)$ & 1.000 \\
\hline Cough & $4(8.9)$ & $3(12.0)$ & 0.694 & $0(0)$ & $0(0)$ & 1.000 \\
\hline Edema peripheral & $2(4.4)$ & $1(4.0)$ & 1.000 & $0(0)$ & $0(0)$ & 1.000 \\
\hline Hypothyroidism & $1(2.2)$ & $1(4.0)$ & 1.000 & $0(0)$ & $0(0)$ & 1.000 \\
\hline Hyperthyroidism & $0(0)$ & $0(0)$ & 1.000 & $0(0)$ & $0(0)$ & 1.000 \\
\hline Neutropenia & 7 (15.6) & $0(0)$ & 0.045 & $0(0)$ & $0(0)$ & 1.000 \\
\hline Alanine aminotransferase increased & $20(44.5)$ & $4(16.0)$ & 0.016 & $3(6.7)$ & $1(4.0)$ & 1.000 \\
\hline Aspertate aminotransferase increased & 15 (33.3) & $3(12.0)$ & 0.050 & $2(4.4)$ & $1(4.0)$ & 1.000 \\
\hline Total bilirubin increased & $5(11.1)$ & $5(20.0)$ & 0.477 & $0(0)$ & $2(8.0)$ & 0.124 \\
\hline Albumin decreased & $4(8.9)$ & $1(4.0)$ & 0.648 & $0(0)$ & $0(0)$ & 1.000 \\
\hline Creatinine increased & $1(2.2)$ & $0(0)$ & 1.000 & $0(0)$ & $0(0)$ & 1.000 \\
\hline
\end{tabular}

AEs, adverse events; HPL, hepatic artery infusion chemotherapy combined with programmed cell death protein-1 inhibitors plus lenvatinib; PL, programmed cell death protein-1 inhibitors plus lenvatinib. 
TABLE 4 | Univariate and multivariate analysis of risk factors for overall survival and progression-free survival.

\begin{tabular}{|c|c|c|c|c|c|c|c|c|c|c|c|c|}
\hline \multirow[t]{3}{*}{ Variables } & \multicolumn{6}{|c|}{ Overall survival } & \multicolumn{6}{|c|}{ Progression-free survival } \\
\hline & \multicolumn{3}{|c|}{ Univariate analysis } & \multicolumn{3}{|c|}{ Multivariate analysis } & \multicolumn{3}{|c|}{ Univariate analysis } & \multicolumn{3}{|c|}{ Multivariate analysis } \\
\hline & HR & $95 \% \mathrm{Cl}$ & $\boldsymbol{P}$ & HR & $95 \% \mathrm{Cl}$ & $P$ value & HR & $95 \% \mathrm{Cl}$ & $P$ & HR & $95 \% \mathrm{Cl}$ & $P$ \\
\hline Age $(y),(</ \geq 50)$ & 1.039 & $0.539-2.003$ & 0.908 & & & & 0.754 & $0.423-1.343$ & 0.338 & & & \\
\hline Gender, (female/male) & 0.413 & $0.413-2.007$ & 0.816 & & & & 1.509 & $0.702-3.240$ & 0.292 & & & \\
\hline Hepatitis B, (no/yes) & 1.194 & $0.516-2.762$ & 0.679 & & & & 1.438 & 0.669-3.089 & 0.352 & & & \\
\hline $\operatorname{AFP}(\mathrm{ng} / \mathrm{ml}),(</ \geq 400)$ & 1.788 & $0.896-3.569$ & 0.099 & & & & 2.096 & $1.144-3.840$ & 0.017 & 2.896 & $1.507-5.568$ & 0.001 \\
\hline PIVKA-II, (mAU/ml), $(</ \geq 400)$ & 1.353 & $0.591-3.097$ & 0.474 & & & & 1.481 & $0.714-3.071$ & 0.291 & & & \\
\hline Liver cirrhosis (no/yes) & 1.225 & $0.476-3.153$ & 0.674 & & & & 1.188 & $0.531-2.660$ & 0.674 & & & \\
\hline Child-Pugh (A/B) & 4.309 & $1.501-12.373$ & 0.007 & 3.709 & $1.239-11.099$ & 0.019 & 2.109 & $0.754-5.897$ & 0.155 & & & \\
\hline $\mathrm{BCLC}(\mathrm{B} / \mathrm{C})$ & 1.163 & $0.475-2.848$ & 0.741 & & & & 1.537 & $0.638-3.701$ & 0.337 & & & \\
\hline Largest tumor size $(\mathrm{cm}),(<10 / \geq 10)$ & 1.670 & $0.837-3.333$ & 0.146 & & & & 1.531 & $0.845-2.773$ & 0.160 & & & \\
\hline Tumor number $(1 />1)$ & 3.127 & $1.097-8.915$ & 0.033 & 3.193 & $1.093-9.327$ & 0.034 & 1.938 & $0.865-4.346$ & 0.108 & & & \\
\hline Tumor distribution (uni-/bi-lobar) & 1.337 & $1.029-1.737$ & 0.030 & & & & 1.322 & $1.062-1.647$ & 0.013 & & & \\
\hline \multicolumn{13}{|l|}{ Tumor thrombus } \\
\hline \multicolumn{13}{|l|}{ Absent } \\
\hline Branch of portal vein & 0.465 & $0.194-1.114$ & 0.086 & & & & 1.054 & $0.487-2.234$ & 0.891 & & & \\
\hline Main portal vein & 0.933 & $0.440-1.979$ & 0.857 & & & & 1.182 & $0.570-2.451$ & 0.653 & & & \\
\hline Extrahepatic metastasis (no/yes) & 1.569 & $0.819-3.003$ & 0.174 & & & & 1.033 & $0.578-1.846$ & 0.912 & & & \\
\hline Treatment (HPL/PL) & 2.770 & $1.437-5.340$ & 0.002 & 3.180 & $1.608-6.290$ & 0.001 & 1.865 & $1.044-3.330$ & 0.035 & 2.702 & $1.440-5.070$ & 0.002 \\
\hline
\end{tabular}

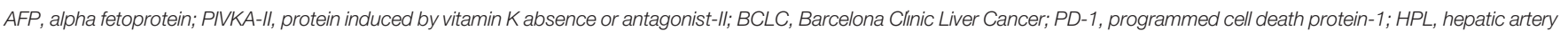
infusion chemotherapy combined with PD-1 inhibitors plus lemvatinb; PL, PD-1 inhibitors plus lenva; HR, hazard rate; Cl, confidence interval.

\section{DISCUSSION}

Treatment strategies for advanced HCC have progressed with the emergence of new tyrosine kinase inhibitors (TKIs) and immune-targeted therapy. Lenvatinib plus pembrolizumab has recently become a potent systemic combination therapy for unresectable HCC (6). In clinical practice, locoregionalsystemic combinations are widely applied due to the overall control of tumor conditions (13). The result of a randomized clinical trial conducted by Ming Shi et al. demonstrated that a combination of sorafenib plus HAIC using FOLFOX agents extends overall survival by $87.5 \%$ or 6.24 months compared to sorafenib alone in HCC patients with portal vein invasion (9). Thus, HAIC may play a role in PL treatment. However, no research has reported the efficacy of HPL versus PL. Our retrospective study demonstrated that in advanced HCC, HPL results in a significantly better survival benefit than PL.

The efficacy benefit observed in the present study may be attributed to the synergistic antitumor effect of PD-1 inhibitors, lenvatinib, and FOLFOX agents. Oxaliplatin induces immunogenic cell death in HCC cells and synergizes with PD1 targeted immunotherapy (14). Lenvatinib inhibits multiple receptor tyrosine kinases (RTKs) targeting VEGFR1-3, FGFR14 , PDGFR $\alpha$, RET, and KIT (15). On the one hand, inhibition of VEGFR and FGFR elicits antitumor immunity and enhances PD-1 checkpoint blockade in HCC (16). On the other hand, antiangiogenesis normalizes tumor vessels and breaks the hypoxic microenvironment of tumors, thereby attenuating the activity of chemoresistance (17-19).

In this study, the median OS and PFS were 8.6 months and 5.4 months in the PL group, respectively, which were better than those observed in a sorafenib monotherapy trial in the Asia-
Pacific region (20). However, the survival outcomes were far worse than those in the Keynote-524 trial (6). Worldwide trials of PD-1 inhibitors or lenvatinib monotherapy in advanced HCC have shown a better OS over 1 year $(21,22)$. Compared to these studies, the patients included in our study were relatively more late-staged with the majority of the patients in the PL group classified with BCLC stage C (88\%), major (48\%) or branch (24\%) of portal vein tumor thrombus, extrahepatic metastases (52\%) and tumor burden over $10 \mathrm{~cm}(60 \%)$. In contrast, the median OS and PFS were significantly better in the homogeneous patients in the HPL group, implying efficacy for the HPL therapy.

The treatment response showed significantly higher ORR and DCR in the HPL group compared to the PL group. Of note, eight patients were unable to assess tumor response in the PL group. One unavoidable reason was that patients treated with systemic medications were not hospitalized, causing the relatively high rate of missed imaging examinations during the treatment, which affected the accurate assessment of tumor response rates. Thus, this variable needs to be further controlled in prospective studies.

In the subgroup analysis, significant differences were not reached in certain subgroups with small proportional cohorts due to limitations in the number of cases. In general, HPL versus PL provided a survival advantage in patients with multiple tumors and tumor diameters greater than $10 \mathrm{~cm}$, but HPL was less effective in patients with main portal vein tumor thrombus and extrahepatic metastases. These findings suggested that HAIC, as a locoregional approach, has a great ability to control intrahepatic lesions but that it may fail to manage extrahepatic metastases. Univariate and multivariate Cox regression analyses showed different factors associated with OS and PFS. This may be partly due to the incongruity between progression and 
survival in the combination therapy of advanced HCC. Patients with the progressive disease could receive more treatment and get inconsistent survival benefit. Of note, HPL was an independent prognostic indicator for both OS and PFS, which confirmed the positive efficacy of HAIC in the combination therapy of PL.

The present study had some limitations. First, the study was a retrospective study in a single center, resulting in inevitable selection bias. Second, the PD-1 inhibitors were varied, which influenced the uniformity of the treatment procedure. Third, the number of cases was relatively small. Findings from this study should be further expanded to a multicenter study to obtain higher-level medical evidence.

Based on our results, HPL is associated with a significantly better treatment response and survival benefits compared to PL. Thus, HPL may be a potential new treatment option for advanced HCC.

\section{DATA AVAILABILITY STATEMENT}

The original contributions presented in the study are included in the article/Supplementary Material. Further inquiries can be directed to the corresponding authors.

\section{REFERENCES}

1. Bray F, Ferlay J, Soerjomataram I, Siegel RL, Torre LA, Jemal A. Global cancer statistics 2018: GLOBOCAN estimates of incidence and mortality worldwide for 36 cancers in 185 countries. CA Cancer J Clin (2018) 68(6):394-424. doi: $10.3322 /$ caac. 21492

2. European Association for the Study of the Liver. Electronic address, e.e.e. and L. European Association for the Study of the, EASL Clinical Practice Guidelines: Management of hepatocellular carcinoma. J Hepatol (2018) 69 (1):182-236. doi: 10.1016/j.jhep.2018.03.019

3. Kudo M, Finn RS, Qin S, Han KH, Ikeda K, Piscaglia F, et al. Lenvatinib versus sorafenib in first-line treatment of patients with unresectable hepatocellular carcinoma: a randomised phase 3 non-inferiority trial. Lancet (2018) 391(10126):1163-73. doi: 10.1016/S0140-6736(18)30207-1

4. Zhu AX, Finn RS, Edeline J, Cattan S, Ogasawara S, Palmer D, et al. Pembrolizumab in patients with advanced hepatocellular carcinoma previously treated with sorafenib (KEYNOTE-224): a non-randomised, open-label phase 2 trial. Lancet Oncol (2018) 19(7):940-52. doi: 10.1016/ S1470-2045(18)30351-6

5. Finn RS, Ryoo BY, Merle P, Kudo M, Bouattour M, Lim HY, et al. Pembrolizumab As Second-Line Therapy in Patients With Advanced Hepatocellular Carcinoma in KEYNOTE-240: A Randomized, DoubleBlind, Phase III Trial. J Clin Oncol (2020) 38(3):193-202. doi: 10.1200/ JCO.19.01307

6. Finn RS, Ikeda M, Zhu AX, Sung MW, Baron AD, Kudo M, et al. Phase Ib Study of Lenvatinib Plus Pembrolizumab in Patients With Unresectable Hepatocellular Carcinoma. J Clin Oncol (2020) 38(26):2960-70. doi: 10.1200/JCO.20.00808

7. Lyu N, Lin Y, Kong Y, Zhang Z, Liu L, Zheng L, et al. FOXAI: a phase II trial evaluating the efficacy and safety of hepatic arterial infusion of oxaliplatin plus fluorouracil/leucovorin for advanced hepatocellular carcinoma. Gut (2018) 67 (2):395-6. doi: 10.1136/gutjnl-2017-314138

8. Ikeda M, Shimizu S, Sato T, Morimotod M, Kojima Y, Inaba Y, et al. Sorafenib plus hepatic arterial infusion chemotherapy with cisplatin versus sorafenib for advanced hepatocellular carcinoma: randomized phase II trial. Ann Oncol (2016) 27(11):2090-6. doi: 10.1093/annonc/mdw323

\section{ETHICS STATEMENT}

This study was conducted according to the ethical guidelines of the 1975 Declaration of Helsinki. The analysis of the patient data was reviewed and approved by the Institutional Review Board and Human Ethics Committee at the Sun Yat-sen University Cancer Center (SYSUCC; Guangzhou, China).

\section{AUTHOR CONTRIBUTIONS}

R-PG, WW, and S-HL designed the study. JM and Y-HT collected the data. JM, Y-HT, MS, WW, S-HL, and R-PG analyzed and interpreted the data. LZ performed the radiological evaluation. JM, Y-HT, S-HL, and R-PG prepared the final draft. All authors contributed to the article and approved the submitted version.

\section{SUPPLEMENTARY MATERIAL}

The Supplementary Material for this article can be found online at: https://www.frontiersin.org/articles/10.3389/fonc.2021. 618206/full\#supplementary-material

9. He M, Li Q, Zou R, Shen J, Fang W, Tan G, et al. Sorafenib Plus Hepatic Arterial Infusion of Oxaliplatin, Fluorouracil, and Leucovorin vs Sorafenib Alone for Hepatocellular Carcinoma With Portal Vein Invasion: A Randomized Clinical Trial. JAMA Oncol (2019) 5(7):953-60. doi: 10.1001/ jamaoncol.2019.0250

10. Bruix J, Reig M, Sherman M. Evidence-Based Diagnosis, Staging, and Treatment of Patients With Hepatocellular Carcinoma. Gastroenterology (2016) 150(4):835-53. doi: 10.1053/j.gastro.2015.12.041

11. Li S, Mei J, Wang Q, Guo Z, Lu L, Ling Y, et al. Postoperative Adjuvant Transarterial Infusion Chemotherapy with FOLFOX Could Improve Outcomes of Hepatocellular Carcinoma Patients with Microvascular Invasion: A Preliminary Report of a Phase III, Randomized Controlled Clinical Trial. Ann Surg Oncol (2020) 27(13):5183-90. doi: 10.1245/s10434020-08601-8

12. Llovet JM, Lencioni R. mRECIST for HCC: Performance and novel refinements. J Hepatol (2020) 72(2):288-306. doi: 10.1016/j.jhep.2019.09.026

13. Palmer DH, Malagari K, Kulik LM. Role of locoregional therapies in the wake of systemic therapy. J Hepatol (2020) 72(2):277-87. doi: 10.1016/ j.jhep.2019.09.023

14. Zhu H, Shan Y, Ge K, Lu J, Kong W, Jia C. Oxaliplatin induces immunogenic cell death in hepatocellular carcinoma cells and synergizes with immune checkpoint blockade therapy. Cell Oncol (Dordr) (2020) 43(6):1203-14. doi: 10.1007/s13402-020-00552-2

15. Yamamoto Y, Matsui J, Matsushima T, Obaishi H, Miyazaki K, Nakamura K, et al. Lenvatinib, an angiogenesis inhibitor targeting VEGFR/FGFR, shows broad antitumor activity in human tumor xenograft models associated with microvessel density and pericyte coverage. Vasc Cell (2014) 6:18. doi: 10.1186/ 2045-824X-6-18

16. Deng H, Kan A, Lyu N, Mu L, Han Y, Liu L, et al. Dual Vascular Endothelial Growth Factor Receptor and Fibroblast Growth Factor Receptor Inhibition Elicits Antitumor Immunity and Enhances Programmed Cell Death-1 Checkpoint Blockade in Hepatocellular Carcinoma. Liver Cancer (2020) 9 (3):338-57. doi: 10.1159/000505695

17. Chung AS, Lee J, Ferrara N. Targeting the tumour vasculature: insights from physiological angiogenesis. Nat Rev Cancer (2010) 10(7):505-14. doi: 10.1038/ nrc2868 
18. Zhang J, Li H, Huangd Z, He Y, Zhou X, Huang T, et al. Hypoxia attenuates Hsp90 inhibitor 17-DMAG-induced cyclin B1 accumulation in hepatocellular carcinoma cells. Cell Stress Chaperones (2016) 21(2):339-48. doi: 10.1007/ s12192-015-0664-2

19. Flamant L, Roegiers E, Pierre M, Hayez A, Sterpin C, De Backer O, et al. TMEM45A is essential for hypoxia-induced chemoresistance in breast and liver cancer cells. BMC Cancer (2012) 12:391. doi: 10.1186/1471-2407-12-391

20. Cheng AL, Kang YK, Chen Z, Tsao CJ, Qin S, Kim JS, et al. Efficacy and safety of sorafenib in patients in the Asia-Pacific region with advanced hepatocellular carcinoma: a phase III randomised, double-blind, placebo-controlled trial. Lancet Oncol (2009) 10(1):25-34. doi: 10.1016/S1470-2045(08)70285-7

21. Kim JJ, McFarlane T, Tully S, Wong WWL. Lenvatinib Versus Sorafenib as First-Line Treatment of Unresectable Hepatocellular Carcinoma: A CostUtility Analysis. Oncologist (2019) theoncologist.2019-0501. doi: 10.1634/ theoncologist.2019-0501
22. Yau T, Hsu C, Kim TY, Choo SP, Kang YK, Hou MM, et al. Nivolumab in advanced hepatocellular carcinoma: Sorafenib-experienced Asian cohort analysis. J Hepatol (2019) 71(3):543-52. doi: 10.1016/j.jhep.2019.05.014

Conflict of Interest: The authors declare that the research was conducted in the absence of any commercial or financial relationships that could be construed as a potential conflict of interest.

Copyright (C) $2021 \mathrm{Mei}$, Tang, Wei, Shi, Zheng, Li and Guo. This is an open-access article distributed under the terms of the Creative Commons Attribution License (CC BY). The use, distribution or reproduction in other forums is permitted, provided the original author(s) and the copyright owner(s) are credited and that the original publication in this journal is cited, in accordance with accepted academic practice. No use, distribution or reproduction is permitted which does not comply with these terms. 